\title{
Asp-15-A stationary device for the measurement of the optical water properties at the NT200 neutrino telescope site
}

\author{
A. Avrorin ${ }^{\text {a }}$, V. Aynutdinov ${ }^{\mathrm{a}}, \mathrm{I}$. Belolaptikov ${ }^{\mathrm{c}}, \mathrm{D}$. \\ A. Yagunov ${ }^{\text {b }}$, A. Zagorodnikov ${ }^{\text {b }}$, V. Zhukov ${ }^{\mathrm{a}}$ \\ a Institute for Nuclear Research, Moscow, Russia \\ ${ }^{\mathrm{b}}$ Applied Physics Institute of Irkutsk State University, Gagarin blvd. 20, Irkutsk 664003, Russia \\ ' Joint Institute for Nuclear Research, Dubna, Russia \\ d Skobeltsyn Institute of Nuclear Physics MSU, Moscow, Russia \\ e DESY, Zeuthen, Germany \\ ${ }^{\mathrm{f}}$ Nizhni Novgorod State Technical University, Nizhni Novgorod, Russia \\ ${ }^{g}$ St.Petersburg State Marine University, St.Petersburg, Russia
} G. Domogatsky ${ }^{a}$, A. Doroshenko ${ }^{a}$, A. Dyachok ${ }^{b}, Z^{2 h} .-A$. Dzhilkibaev ${ }^{a}$, S. Fialkovsky ${ }^{f}$, O. Gaponenko $^{a}$, K. Golubkov ${ }^{\text {a }}$, O. Gress ${ }^{\text {b }}$, T. Gress ${ }^{\text {b }}$, O. Grishin ${ }^{\text {b }}$, V. Karnaukhov ${ }^{\text {d }}$, A. Klabukov ${ }^{\text {a }}$, K. Konischev ${ }^{c}$, A. Koshechkin ${ }^{\text {a }}$, F. Koshel ${ }^{\text {a }}$, A. Korobchenko ${ }^{b}$, V. Kulepov ${ }^{f}$, D. Kuleshov ${ }^{\text {a }}$, L. Kuzmichev ${ }^{\text {, }}$, V. Lyashuk ${ }^{\text {, }}$,

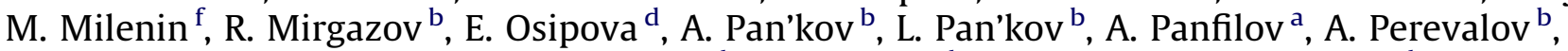
D. Petukhov ${ }^{\mathrm{a}}$, E. Pliskovsky ${ }^{\mathrm{c}}$, V. Poleschuk ${ }^{\mathrm{b}}$, A. Rastegin ${ }^{\mathrm{b}}$, M. Rozanov $^{\mathrm{g}}$, V. Rubtzov $^{\mathrm{b}}$, E. Ryabov $^{\mathrm{b}}$,

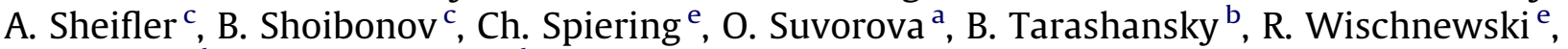

\section{A R T I C L E I N F O}

\section{Article history:}

Received 5 October 2010

Received in revised form

14 June 2012

Accepted 14 June 2012

Available online 30 June 2012

Keywords:

Neutrino telescope

Neutrino astronomy

UHE neutrino

BAIKAL

\begin{abstract}
A B S T R A C T
The operation of large underwater neutrino telescopes requires the precise knowledge of the water parameters governing light absorption and scattering, as well as a continuous monitoring of these parameters. For this purpose, a stationary underwater device, ASP-15, has been developed by the Baikal collaboration. We describe the basic assumptions and formulae behind ASP-15, the methods how absorption length, scattering length and phase functions are determined, the design of the device, and give some results obtained over many years of operation in conjuction with the Baikal telescope NT200.
\end{abstract} (c) 2012 Elsevier B.V. All rights reserved.

\section{Introduction}

The propagation of light in water is governed by two basic phenomena: absorption and scattering. In the first case the photon is lost, in the second case it changes its direction. For many practical and scientific applications, a precise knowledge of the parameters characterizing both processes is essential. One of these cases is the construction and operation of underwater neutrino telescopes in lakes or oceans.

The exploitation of deep natural water basins for the detection of high energy cosmic neutrinos was proposed 50 years ago [1]. A deep underwater neutrino detector consists of a lattice of

\footnotetext{
* Corresponding author. Tel./fax: + 73952332140.

E-mail address: nbudnev@api.isu.ru (N. Budnev).
}

photomultipliers which record the Cherenkov light from charged secondary particles generated in neutrino interactions. First practical approaches have been the DUMAND project close to Hawaii [2] and the Baikal project in the Siberian Lake Baikal [3]. It was the Baikal project which operated a first stationary underwater configuration of photomultipliers in 1984 and 1986 [4,5].

After several stages of growing complexity [3,6], in 1998 a first full-scale neutrino telescope NT200 [7,8] was put into operation in Lake Baikal. It is installed at a depth of $1.1 \mathrm{~km}, 3.6 \mathrm{~km}$ from shore, and consists of 192 photomultipliers which are housed in pressure-resistant glass spheres arranged on eight vertical strings. The overall height of the configuration is $72 \mathrm{~m}$, its diameter $42 \mathrm{~m}$. At present, the Baikal collaboration works towards a cubic kilometer telescope BAIKAL-GVD in Lake Baikal [9].

Nowadays, ANTARES [10] is another underwater neutrino telescope operating in the Mediterranean Sea. Also the three 
Mediterranean neutrino projects ANTARES, NEMO [11] and NESTOR [12] have joined their forces in preparation of a neutrino telescope at the cubic kilometer scale, KM3NeT [13].

Before they reach the photomultipliers, the photons generated by charged particles propagate over distances which may considerably exceed the absorption length and the scattering length of light. This means that the charged particle detection efficiency strongly depends on the water parameters $[14,15]$. Therefore, the interpretation of the experimental data taken with a neutrino telescope requires a reasonably precise knowledge of these parameters. The inherent optical properties (IOPs) generally chosen as a measure for absorption and scattering phenomena depending on a light wavelength $\lambda$ in natural water are: (I) the absorption coefficient spectrum $a(\lambda)$ (or the absorption length $L_{a}=1 / a$ ), (II) the scattering coefficient spectrum $b(\lambda)$ (or the scattering length $L_{b}=1 / b$ ), (III) the attenuation coefficient spectrum $c(\lambda)=a(\lambda)+b(\lambda)$ (or the attenuation length $L_{c}=1 / c$ ), (IV) the scattering phase function $\chi(\gamma, \lambda)$ (also referred to as the volume scattering function) [16], where $\gamma$ is the scattering angle. Since the values of the IOPs in natural water vary with time, a long-term in situ monitoring of IOPs is mandatory to operate a neutrino telescope. The measurement of the IOPs is also one of the central tasks in physical oceanology and limnology: light absorption and scattering effects in water can provide important information on the concentration of dissolved organic matter and suspended forms of organic matter, their mutual transformation, the rate of sedimentation, as well as on other important biological and physical processes in natural basins.

A lot of different instruments - commercial and custom designed -have been constructed for the measurement of optical water parameters. Most of them use short transmission cells with a fixed length of the light path (see e.g. [17] and references therein). These techniques require careful calibration procedures with pure water or nitrogen before each measurement in order to evaluate the cell characteristics. They are therefore not well suitable for a long-term operation under water. This is the motivation to develop new devices and methods for monitoring the IOPs of water at the sites of neutrino telescopes. In order to avoid the necessity of knowing precisely the optical parameters of the device, the light path must be variable. In this case, signal amplitude versus distance can be measured and attenuation parameters determined by a fit. To our knowledge, the only attempts to build such a system are described in [18-21]. In these cases, however, only 3 or 4 fixed adjustable distances were possible, and changes could not be made remotely but had to be made at the surface. This prevents any application for long-term measurements.

We have constructed an instrument for long-term in situ monitoring of the inherent optical properties of deep water, ASP-15. The acronym stands for Absorption, Scattering and Phase function meter, working at 15 wavelengths of visible light. ASP-15 can be either operated from the ice cover of Lake Baikal (nonstationary mode, during winter expedition), or installed on stationary, moored buoy-stations (stationary mode, in conjuction with the Baikal neutrino telescope), thus operating remotely controlled from the shore control station for one year.

In the following, we first describe the methods to measure absorption coefficient (Section 2), scattering coefficient (section 3) and phase function (Section 4). The ASP-15 device is described in Section 5. Measurement procedures are sketched in Section 6 and errors discussed in Section 7. Section 8 gives examples of results, Section 9 a summary.

\section{Determination of the absorption coefficient}

The study of the IOPs with ASP-15 is based on the measurement of the light field of a point-like isotropic source with time-independent intensity. The light field is characterized by the differential flux of photons defined as $B(\vec{R}, \vec{\Omega})=d N / d S d \vec{\Omega}$, where $d N$ is the number of photons, passing an area $d S$ at a spatial point with coordinate $\vec{R}$ in the direction $\vec{\Omega}(\theta, \varphi)$ within a solid angle $d \vec{\Omega}=\sin \theta d \theta d \varphi$. For a point-like isotropic source the differential flux depends only on the distance $R=|\vec{R}|$ from the source and the angle $\theta$ between the direction vector $\vec{\Omega}$ and the coordinate vector $\vec{R}: \cos \theta=(\vec{\Omega} \vec{n})$, where $\vec{n}=\vec{R} / R$.

Let us define $N^{\circ}(R), N_{1}(R)$ and $N_{2}(R)$ as:

$N^{\circ}(R)=\int_{0}^{2 \pi} d \varphi \int_{0}^{\pi} B(R, \theta) \sin \theta d \theta$,

$N_{1}(R)=\int_{0}^{2 \pi} d \varphi \int_{0}^{\pi / 2} B(R, \theta) \cos \theta \sin \theta d \theta$,

$N_{2}(R)=-\int_{0}^{2 \pi} d \varphi \int_{\pi / 2}^{\pi} B(R, \theta) \cos \theta \sin \theta d \theta$

Here, $N^{\circ}(R)$ is the spatial illuminance at distance $R$ from a source, which is derived from an integration of the differential flux $B(\vec{R}, \vec{\Omega})$ over all directions of photons; $N_{1}(R) d \vec{\sigma}$ and $N_{2}(R) d \vec{\sigma}$ are the numbers of photons, leaving and entering the spherical surface with radius $R$ through an elementary fixed area $d \vec{\sigma}$ (the orientation of $d \vec{\sigma}$ is given by the vector $\vec{n}=\vec{R} / R$ ).

Given the photon current through a spherical surface $S(R)=$ $4 \pi R^{2}$ :

$$
\begin{aligned}
J(R) & =4 \pi R^{2} \int_{0}^{2 \pi} d \varphi \int_{0}^{\pi} B(R, \theta) \cos \theta \sin \theta d \theta \\
& =4 \pi R^{2}\left(N_{1}(R)-N_{2}(R)\right),
\end{aligned}
$$

the number of photons which are absorbed in the volume $d V=4 \pi R^{2} d R$ between two spherical surfaces $S(R)$ and $S(R+d R)$ around the light source, has to be equal to the difference of photon currents through $S(R+d R)$ and $S(R)$ :

$d J(R)=-a d V \int_{0}^{2 \pi} d \varphi \int_{0}^{\pi} B(R, \theta) \sin \theta d \theta$,

where $a$ is the absorption coefficient of light in water. Taking into account Eqs. (1)-(4), the following expression for the absorption coefficient $a$ can be derived from Eq. (5) $[21,22,23]$ :

$a=-\frac{1}{N^{\circ}(R) R^{2}} \frac{d\left(\left[N_{1}(R)-N_{2}(R)\right] R^{2}\right)}{d R}$.

So, to determine the absorption coefficient $a$, one has to measure three photometric characteristics, as a function of $R: N_{1}(R), N_{2}(R)$ and $N^{\circ}(R)$. Some approximations are applicable to make this method more suitable for practical use. In the first place, for water with a strongly forward-peaked scattering phase function $\chi(\gamma)$ as typical for clean sea waters and Lake Baikal, one has:

$\mu(R)=\left[N_{1}(R)-N_{2}(R)\right] / N^{\circ}(R) \approx 1$.

We checked the relation (7) by Monte-Carlo methods for different values of absorption and scattering coefficients and forwardpeaked phase scattering functions and also measured $\mu(R)$ in Lake Baikal. In both cases, the relation (7) turns out to be correct within $1 \%$ as long as $R<L_{a}, L_{b}$ [24]. Usually, also the following condition turned out to be applicable in natural water:

$d\left[N_{1}(R) R^{2}\right] / d R \gg d\left[N_{2}(R) R^{2}\right] / d R$.

It is violated in media with $L_{a} \gg L_{b}$ and isotropic scattering, especially at small distances. For typical sea waters it holds, and the absorption coefficient $a$ can be determined by simplifying 


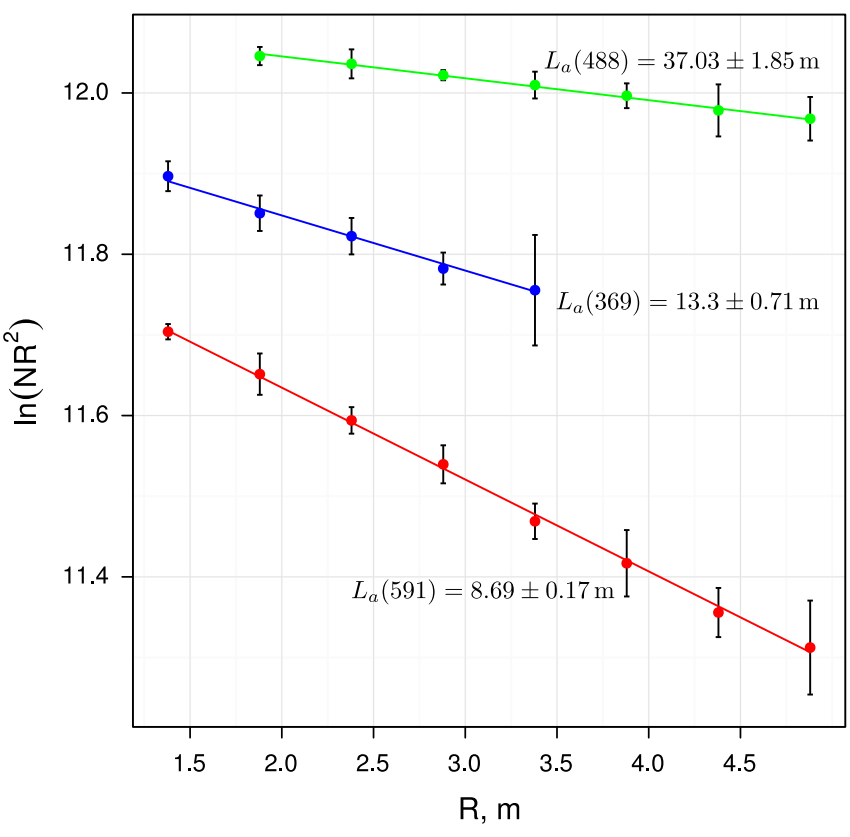

Fig. 1. The measured dependence of $\ln \left(N R^{2}\right)$ on distance $R$, for three different wavelengths.

expression (6) to

$a \approx-\frac{1}{N_{1}(R) R^{2}} \frac{d\left[N_{1}(R) R^{2}\right]}{d R}=-\frac{d \ln \left[N_{1}(R) R^{2}\right]}{d R}$

and measuring only $N_{1}(R)$.

With the ASP-15 instrument, we have carried out some thousand measurements of the absorption coefficient of water at various wavelengths in the visible range. Usually, an excellent agreement of experimental data with the exponential law behavior of the $N_{1}(R) R^{2}$ according to the formula (9) is observed (see the examples in Fig. 1). It proves the applicability of the method as well as the quality of the data. Each fit of the data sets in Fig. 1 corresponds to one point of the absorption spectrum (examples in Fig. 6).

\section{Determination of the scattering coefficient}

This method is the logical continuation of the previous one. For the same conditions and approximations as in the previous part the total number of photons $N_{1}$ (let us denote it $N_{t}$ ) from an isotropic point-like source hitting the unit area of a detector (Fig. 2a) is:

$N_{t}(R) \approx \frac{N_{0}}{4 \pi R^{2}} \exp (-a R)$

where $N_{0}$ is a source intensity.

The number of photons $N_{t}$ is added up from two samples of photons, which reach the detector. The first sample consists of direct photons, which were not scattered before hitting the detector. Their number $N_{d}$ decreases with distance from the source as:

$N_{d}(R)=\frac{N_{0}}{4 \pi R^{2}} \exp (-c R)$

where $c=a+b$. Photons from the second sample were scattered before they hit the detector. Placing a screen with diameter $D$ at a fixed distance $r \ll R$ from the isotropic, point-like $(d \ll D)$ source (Fig. 2c), one can measure the number $N_{s}$ of those scattered
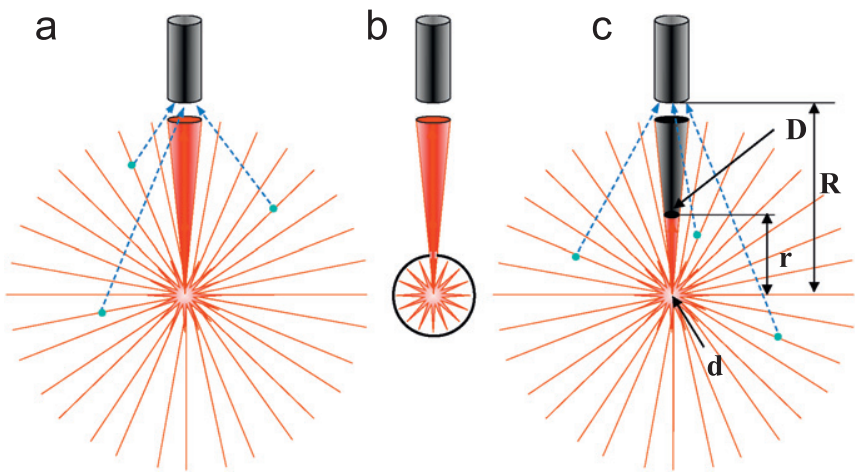

Fig. 2. Illustration of the method to determine the scattering coefficient.

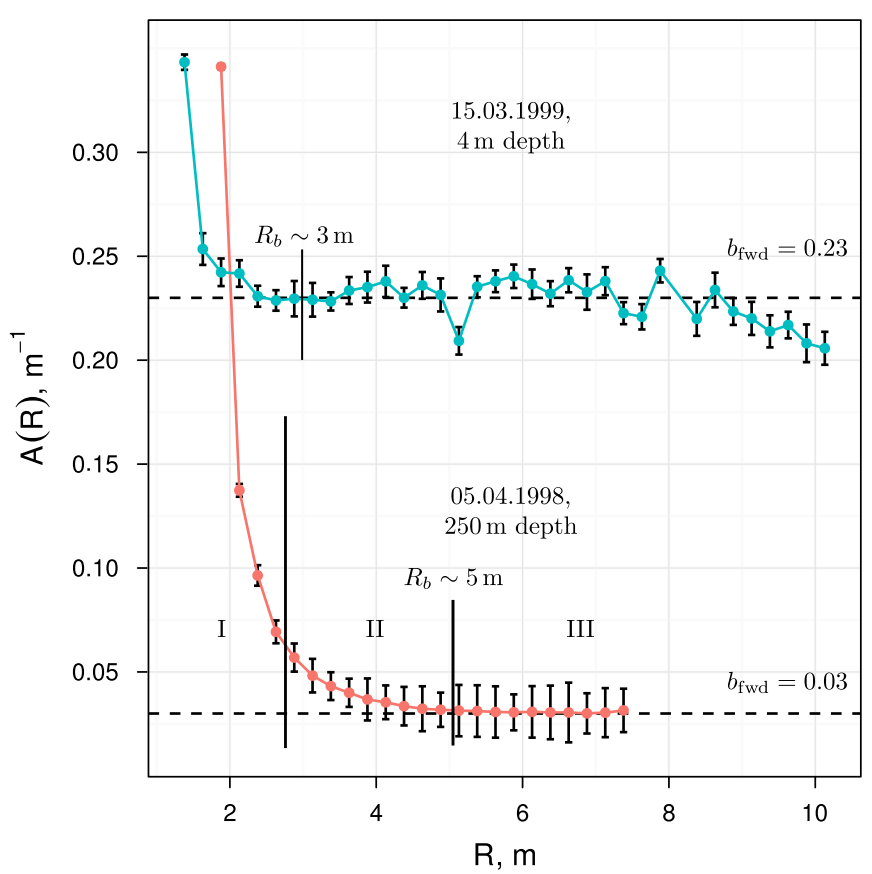

Fig. 3. Dependence of the value $A(R)$ on the distance $R$ between source (shadowed by a screen) and detector, at depths $4 \mathrm{~m}$ and $250 \mathrm{~m}$.

photons:

$N_{s}(R) \approx N_{t}-N_{d}=\frac{N_{0}}{4 \pi R^{2}}[\exp (-a R)-\exp (-c R)]$.

Measured $N_{s}$ and $N_{t}$, the value of the scattering coefficient $b$ can be estimated as:

$b \approx-\frac{\ln \left(1-N_{s} / N_{t}\right)}{R}$.

So, we do not need to know $N_{0}$, which cannot be kept constant over times typical for stationary mooring. In reality, apart from scattering, diffraction at the edge of the screen will contribute additional photons hitting the detector. In practice the onset of diffraction can easily be determined by moving source and screen with respect to the detector. We have measured $N_{s}(R)$ and $N_{t}(R)$ at different distances $R$ and then calculate the value

$A(R)=-\frac{\ln \left(1-N_{s}(R) / N_{t}(R)\right)}{R}$.

Fig. 3 shows the dependence of $A(R)$ on $R$, measured at two different depths. The three ranges (1), (2) and (3) as indicated in Fig. 3 correspond to direct light (as defined by simple geometry), dominance of diffracted light, and scattered light, respectively. Note that at $4 \mathrm{~m}$ depth scattering is so strong, that scattered light 
overshines any diffraction contribution as soon as the direct-light region is left. At greater depth, scattering is smaller and the $R$-range of diffraction dominance is clearly identifiable. In other words: $A(R)$ (Eq. (14)) equals $b$ (Eq. (13)) at distance $R>R_{b}$, where $A$ is constant. $R_{b}$ increases with decreasing $b$ (compare the curves for 4 and $250 \mathrm{~m}$ ).

The method requires $d \ll D \ll r \ll R$ (see Fig. 2c). In ASP15 these parameters have the following values: $d=0.08 \mathrm{~cm}, D=0.3 \mathrm{~cm}$, $r=30 \mathrm{~cm}$ and $R$ varies from $R=0.5 \mathrm{~m}$ up to $R=15 \mathrm{~m}$. The screen shades photons emitted by the source within a very small solid angle $\delta \Omega=7.85 \times 10^{-5} \mathrm{sr}$. Since this solid angle is larger than the light sensor acceptance, some fraction of $N_{s}$ (i.e. the scattering of those photons hitting the screen) is missing in the measurement. This loss is, however, negligible even for strongly forward-peaked phase functions with average cosine $\overline{\cos \gamma}=0.95$ and does not in any substantial way affect the determination of the scattering coefficient $b$. The scattering coefficient may be represented as the sum of the forward and backward scattering coefficients $b=b_{\mathrm{fwd}}+b_{\text {back }}$, where:

$b_{\mathrm{fwd}}=2 \pi b \int_{0}^{\pi / 2} \chi(\gamma) \sin (\gamma) d \gamma$

$b_{\text {back }}=2 \pi b \int_{\pi / 2}^{\pi} \chi(\gamma) \sin (\gamma) d \gamma$

and the scattering phase function $\chi(\gamma)$ satisfies the following normalization condition:

$\int_{4 \pi} \chi(\Omega) d \Omega=2 \pi \int_{0}^{\pi} \chi(\gamma) \sin (\gamma) d \gamma=1$

Results of MC simulations show that for the case of a point-like isotropic source and strongly anisotropic phase functions the flux of photons at distance $R$ from the source is formed mainly by the photons which were emitted within a cone with half angle about 30-60 around $\vec{R}$. This means that our estimation of $b$ coincides with $b_{\text {fwd }}$ within the experimental errors. An estimation of $b_{\text {back }}$ can be derived from the measured value of $b_{\mathrm{fwd}} \simeq b$ and from the value of the asymmetry coefficient of the scattering phase function $K$, which is defined as

$K=\frac{b_{\text {fwd }}}{b_{\text {back }}}$.

An estimation of $K$ is derived from the ratio $K_{\text {exp }}$ of detected photons which were emitted by unscreened and screened isotropic source, respectively (source 2 in Fig. 5, left panel). It was shown in [26] that $K_{\text {exp }}$ is a good estimation of $K$ with an accuracy of about $15 \%$.

\section{Determination of the scattering phase function}

In the single-scattering approximation, the number of photons $N(\alpha, R) d S d \Omega$ (Fig. 4), hitting a detector area element $d S$ within a solid angle $d \Omega$ at an angle $\alpha$ from the source-detector axis is [25]:

$$
\begin{aligned}
N(\alpha, R) d S d \Omega= & b \frac{N_{0} d S d \Omega}{R \sin \alpha} \int_{0}^{\pi-\alpha} \chi(\alpha+\theta) \\
& \times \exp \left[-c R \frac{\sin \alpha+\sin \theta}{\sin (\alpha+\theta)}\right] d \theta .
\end{aligned}
$$

Here $N_{0}$ is the source intensity, $\chi(\alpha+\theta)$ is the phase scattering function. For small $\alpha$ one can approximate the photon path length by $r_{1}+r_{2} \approx R$ (Fig. 4). Then, from Eq. (15) follows:

$\chi(\alpha) \approx-\frac{R}{b N_{0}} \exp (c R) \frac{d[N(\alpha, R) \sin \alpha]}{d \alpha}$.

Eq. (16) is used for an estimation of the phase scattering function by measuring the number of photons under different angles $\alpha$ (see, Section 8.3).

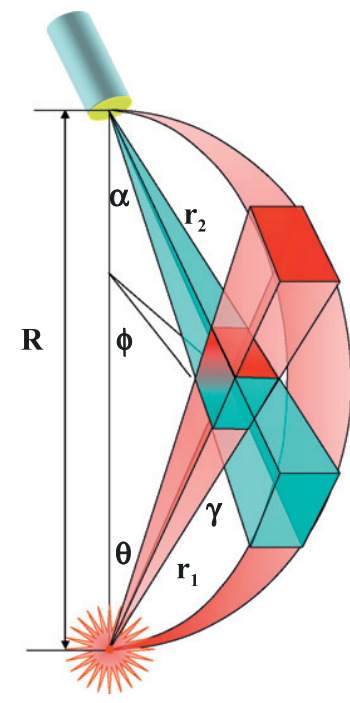

Fig. 4. Definition of angles and distances for single-scattered photons from an isotropic light source.

\section{ASP-15 - an instrument for long-term monitoring of inherent optical properties of natural water}

A sketch of ASP-15 is shown in Fig. 5. The water-tight container which houses the electronics block, two PMTs, light filters and a step motor is made from an aluminum tube with diameter $200 \mathrm{~mm}, 1000 \mathrm{~mm}$ length and a wall thickness of $30 \mathrm{~mm}$. The ends of the tube are closed with two flat aluminum lids with thickness $40 \mathrm{~mm}$. The total weight of the equipped container is about of $60 \mathrm{~kg}$ in air. There are two organic glass illuminators in the middle part of the tube.

Outside of the container, in the water, there are two light receiving channels: channel 1 with a wide aperture which is used for $a$ and $b$ measurements, and channel 2 with a narrow $0.5^{\circ}$ aperture for the measurement of the scattering phase function.

Channel 1 is equipped with a milk glass window with $40 \mathrm{~mm}$ diameter and $5 \mathrm{~mm}$ thickness. This window is followed by a long tube with black inner surface, which limits the aperture for the light hitting the interference filters to $10^{\circ}$.

Channel 2 is equipped with a mirror and a lens hood with $30 \mathrm{~cm}$ length and $15 \mathrm{~mm}$ diameter, which are rotated by a step motor over an angular range of $360^{\circ}$, with a step of $2^{\prime}$. Photons reflected by this mirror pass the glass window, a collimator with an aperture of $0.5^{\circ}$ and a light filter, and eventually hit PMT-2. The lens hood protects the receiver 2 from direct light during the measurement of the scattering phase function.

PMTs of type FEU-130 operating in photon rate counting mode are used as receivers. Non-linearities of the receivers are less than $1 \%$ over a dynamic range up to $10^{5} \mathrm{~Hz}$ and can be remotely controlled by two LEDs with adjustable brightnesses. The PMT dark current is about $30 \mathrm{~Hz}$.

There are 15 interference light filters with bandwidth $3-5 \mathrm{~nm}$ in the range of 350-690 $\mathrm{nm}$. Light filters are arranged in plastic frames and connected to a caterpillar-like structure. A step motor moves the caterpillar which changes the light filters in front of PMT-1 and PMT-2. Also there is an empty frame in the caterpillar, which is used for the measurement of the scattering phase function.

On the outer side of the bottom lid, a step motor with a gear filled with oil is mounted. The motor rotates a block with a strained cable loop of $15 \mathrm{~m}$ length. A light-weight platform mounted on the cable has two light sources and two shadowing 

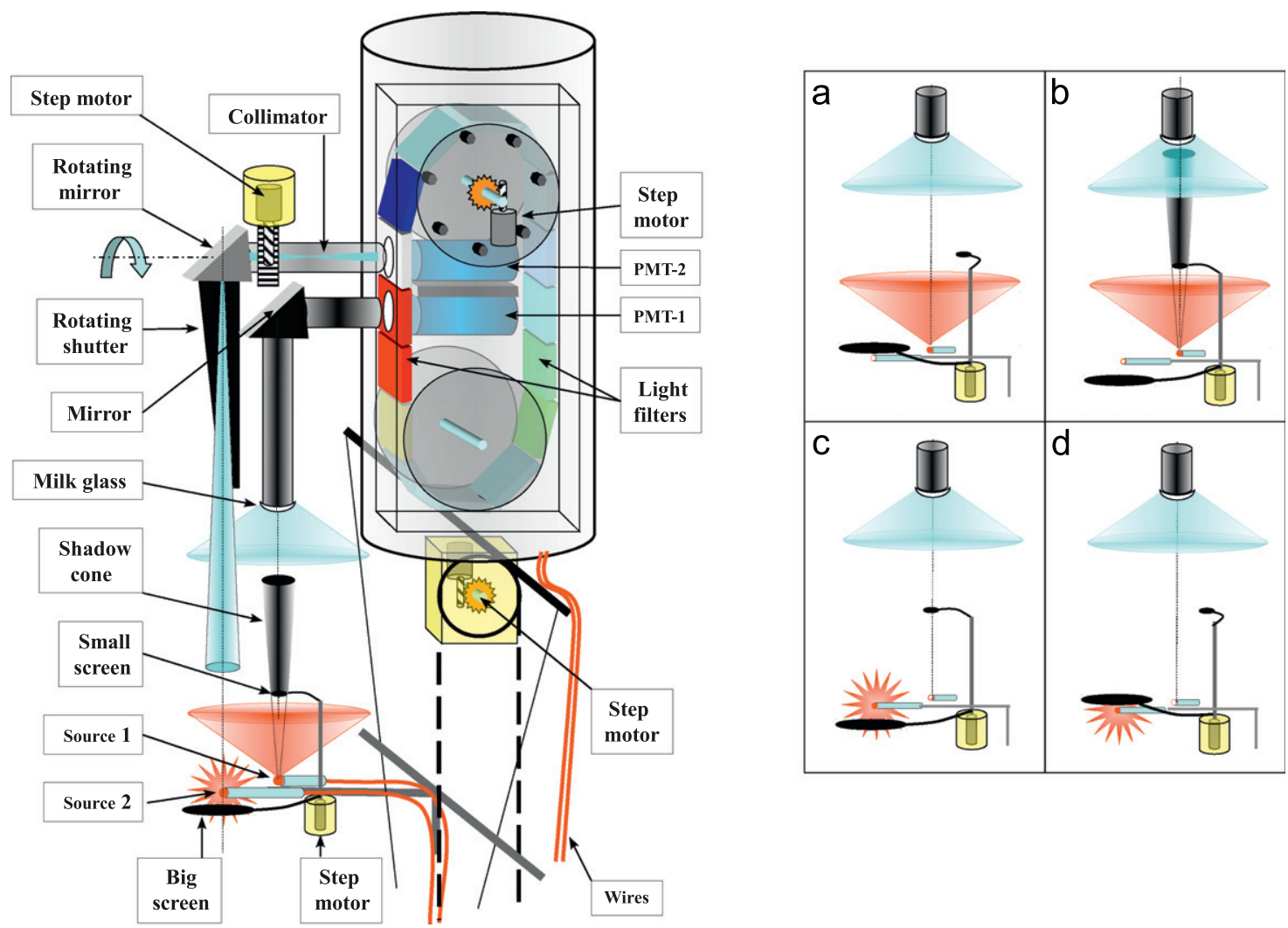

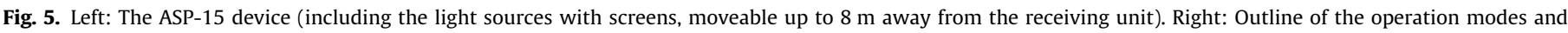
formulas to determine the scattering parameters $b_{\mathrm{fwd}}$ and $b_{\text {back }}$ (see Section 6 ).

screens. Light sources and screens can be moved vertically from $R=0.5 \mathrm{~m}$ to $R=15 \mathrm{~m}$ with respect to the light receiving channels.

Tiny halogen lamps KGMN-27 in hermetical quartz tubes are used as light sources. Supply voltage and current of the lamp are $27 \mathrm{~V}$ and $100 \mathrm{~mA}$, respectively. The optimal lamp brightness depends on the used light filter and is controlled by 8-bit digital-to-analogue converter. It is chosen in a way that the linear dynamic range of the PMT is not exceeded. The electrical current through the lamp is stabilized at a level of $0.01 \%$.

The light source 1 is covered with a light-tight cup with an aperture $d=0.8 \mathrm{~mm}$ through which light is emitted within a cone with a full angle of about $60^{\circ}$. The axis of the light cone is directed towards the center of channel 1.

Between the source 1 and the milk glass window, at a fixed distance $r=30 \mathrm{~cm}$ from the source, a small shadowing screen with a diameter of $D=3 \mathrm{~mm}$ (shadowing angle $0.5^{\circ}$ ) is arranged.

The size of the isotropic light source 2 is about $10 \mathrm{~mm}$. Source 2 can be shadowed by a big screen with a diameter of $3 \mathrm{~cm}$, which is placed at a distance of $3 \mathrm{~mm}$ from the source. Source 2 is located below channel 2. Both screens can be moved simultaneously in the horizontal plane for shading the light sources, as it is shown in Fig. 5 (right panel). The movement of screens is performed by the tiny step motor.

The power is supplied over one wire and the armoring of a $7 \mathrm{~km}$ long multi-core cable on the bottom of Lake Baikal. The voltage of the power supply is $300 \mathrm{~V}$, the maximal current is $300 \mathrm{~mA}$.

The deployment of ASP-15 at a depth of $1000 \mathrm{~m}$ for long-term operation, as well as lifting for preventive maintenance and the performance of methodological and research measurements are made in March-April each year from the ice cover of Lake Baikal. The device is fixed at a cable located $300 \mathrm{~m}$ away from the center of NT200.
Instrument operation, data acquisition and transmission are carried out by a controller and power line modem, developed for NT200. The modem uses PWM (Pulse Width Modulation) for signal transmission via a 7-km geophysical cable to shore. The measurement of optical parameters of the water is performed by the shift operator of NT200 or remotely via the Internet.

\section{Measurement procedures}

\subsection{The absorption spectrum}

To measure the absorption spectrum, the isotropic light source 2 is moving automatically over distances which make the best use of the dynamic range of the device and are specific for each of the light filters. The measurement of $N(R)$ is made at ten distances and $a(\lambda)$ is determined for each filter. During the measurement, the stability of the light source intensity is about $0.1 \%$. This process is remotely performed by the NT200 shift operator at the shore and runs automatically over all filters.

\subsection{The scattering spectrum}

To measure the scattering spectrum, the distance $R_{b}$ (see Fig. 3) has to be determined by evaluating the dependence of $A(R)$ vs. $R$ for each of the light filters.

Then both light sources are moved to a distance $R=R_{b}$, source 1 is turned on, $N_{t}(\lambda)$ (Fig. 5 right, step $A$ ) and $N_{s}(\lambda)$ (Fig. 5 right step $B$ ) are measured and the scattering spectrum is calculated according to Eq. (13). After that, source 1 is turned off and source 2 is turned on, followed by the measurement of the total and the backscattered light from this source (Fig. 5 right, steps $C$ and $D$ ). The series of these four measurements allows estimating 
$b_{\mathrm{fwd}}=-\ln (1-B / A) / R$ and $K=n B / D$, and calculating $b_{\mathrm{back}}=b_{\mathrm{fwd}} / K$. Here the normalization coefficient $n$ is the ratio of source intensities, $n=C / A$, and $A, B, C, D$ are the results of the measurements in the respective steps defined in Fig. 5, right panel. Again, this process is remotely performed by the NT200 shift operator at the shore and runs automatically over all distances and filters.

\subsection{The scattering phase function}

First, the frame without a filter is moved in front of PMT-2. Then, both light sources are moved to their minimum distance to receiver 2 , source 2 is turned on and source 1 is turned off. The instrument is ready to measure the scattering phase function. Then the measurement is done by rotating the mirror in steps of $2^{\prime}$ between $0^{\circ}$ and $360^{\circ}$. This process is performed remotely from shore, but requires the manual control of an expert.

\section{Statistic and systematic errors}

The usual measuring procedure of the absorption coefficient $a$ is as follows. At a fixed position $R_{j}$ of source 1 (Fig. 5 left) we perform a series of 5-10 (depending on the light filter used) of $1 \mathrm{~s}$ long measurements of the photon flux $N_{1}$ (see Section 2). The average value of the variable $\xi_{i}=\ln \left(N_{1} R_{j}^{2}\right)$ and the rms error $\sigma_{\xi_{i}}$ are calculated. $\sigma_{\xi_{i}}$ includes statistical and systematic errors. Systematic errors are defined by uncertainties of the source position $\Delta R_{j}=0.005 \mathrm{~m}$ and the stability of the source intensity which is controlled at a level of $10^{-3}$ during the measurement cycle. Systematic errors of $\xi_{i}$ are at a level of $0.05 \%$ and are negligible compared to the statistical errors $\sim(0.2-0.7) \%$.

Value and error of the absorption coefficient $a$ are derived from a $\chi^{2}$-minimization procedure, using average values of $\xi_{i}$ and $\sigma_{\xi_{i}}$ corresponding to measurements at different positions $R_{j}$ of the source 1 . Measuring uncertainties of the absorption coefficient $a$ are about of $(2-5) \%$.

The measuring procedure of the scattering coefficient $b$ is described in Section 6.2. According to this procedure the value of $b$ is derived from measurements at source 1 position $R_{b}$, where effects of light diffraction are negligible. Series of $1 \mathrm{~s}$ long measurements of $N_{t}$ and $N_{s}$ (see Eq. (13)) have been performed and average values $\overline{N_{t}}$ and $\overline{N_{s}}$, as well as rms errors $\Delta N_{t}, \Delta N_{s}$ have been calculated. The value of the scattering coefficient $b$ is estimated by using $\overline{N_{t}}$ and $\overline{N_{s}}$. The relative error $\delta_{d} b=\Delta b / b$ is estimated as:

$\delta_{d} b \approx \frac{1}{b R_{b}} \sqrt{\left(\frac{\Delta N_{s}}{\bar{N}_{t}}\right)^{2}+\left(\frac{\overline{N_{s}} \Delta N_{t}}{{\overline{N_{t}}}^{2}}\right)^{2}+\left(b \Delta R_{b}\right)^{2}}$.

Typical values of $\delta_{d} b$ cover the range of (3-6)\% depending on the light wavelength.

Our estimation of the methodical errors $\delta_{m}$ of the IOP measurement, with the approximations as described above, but with an "ideal" instrument with an isotropic light source and a wide aperture receiver is [24,26]:

$\delta_{m} a(\lambda) \sim 2 \%$,

$\delta_{m} b_{\mathrm{fwd}}(\lambda) \sim 5 \%$,

$\delta_{m} K(\lambda) \sim 30 \%$,

$\delta_{m} \chi\left(2.5^{\circ}<\gamma<30^{\circ}\right) \sim 10 \%$.

These errors have been obtained by Monte-Carlo calculations, assuming certain input parameters and then checking against the parameters returned by the procedure. Errors are due to various approximations which are not related to imperfect instruments, e.g. those mentioned in the context for formulae (7) and (8). Additional errors for a "real" instrument are due e.g. to the following reasons: the sources are not exactly isotropic; the sources are not point-like; there is some reflection from the big screen. Whereas the former errors can be estimated by MC simulations but cannot be made smaller, the latter must just be kept as small as possible by proper construction of the device. Our estimation of the total errors for the wavelength range of 400-600 nm for the case of ASP-15 yields [24]:

$\delta a(\lambda) \sim 5 \%$,

$\delta b_{\text {fwd }}(\lambda) \sim 10 \%$,

$\delta K(\lambda) \sim 50 \%$,

$\delta \chi\left(2.5^{\circ}<\gamma<30^{\circ}\right) \sim 20 \%$.

For the interpretation of the results from a neutrino telescope and other practical purposes, this is sufficient.

\section{Some exemplary results}

In this section we present some results, which have been obtained with the ASP-15 device at the site of the Baikal Neutrino Telescope NT200. We also refer to a measurement campaign performed in March/April 2001 [29] where ASP-15 has taken data simultaneously with the commercial device AC9 [17]. The values of absorption and scattering coefficients $a(\lambda)$ and $b(\lambda)$ measured with both instruments turned out to be identical within errors.

\subsection{Absorption}

Usually, absorption spectra are rather smooth and reproducible (Fig. 6, data from April 4, 2001). At large wavelengths, the spectra are close to that of pure water and the cleanest ocean water. The difference for blue light is due to dissolved organic matter ("yellow matter"), which is responsible for the absorption of light in this part of the spectrum. Its concentration in Lake Baikal is much higher than in deep ocean waters. The typical value of the absorption coefficient for $\lambda=488 \mathrm{~nm}$ (the minimum of the absorption spectrum) is $0.045 \mathrm{~m}^{-1}$ at $1000 \mathrm{~m}$ depth (corresponding to an absorption length of $22.2 \mathrm{~m}$ ).

Over many years, an absorption length of $22.2 \mathrm{~m}$ has turned out to be reproducible within $(10-15) \%$, as shown in Fig. 7. However, in some rare cases, the absorption spectra strongly change over a few days (see the curves for April 4 and 8 in Fig. 6) and even show narrow extrema at definite wavelengths (May 4). Since as a rule in these periods the linear behaviour $\ln \left(N(R, \lambda) R^{2}\right)$ vs. $R$ is violated, we assign these results a lower credibility. The systematic errors given above apply only to cases of strictly exponential behaviour.

\subsection{Scattering}

The variation in light scattering in the forward hemisphere in Lake Baikal is much stronger than that of absorption. It changes with depth and time. At a given depth, variations in time may reach an order of magnitude. Fig. 8 shows the depth dependence of the forward scattering coefficient, $b_{\mathrm{fwd}}$, for $\lambda=488 \mathrm{~nm}$ as measured at March 3, 2000. The profile changes from year to year, but the maximum gradient is always over the first $50 \mathrm{~m}$ of depth. In the deep zone (500-1100 m), $b_{\text {fwd }}$ usually decreases by about a factor of 2 , followed by a small increase close to the bottom of the lake. 


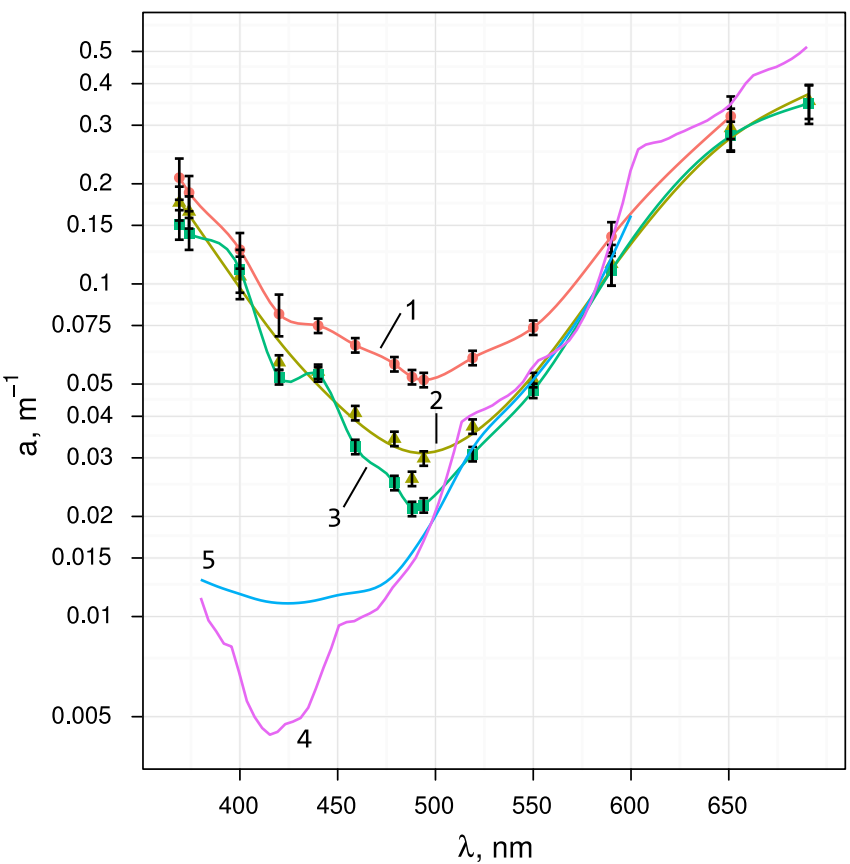

Fig. 6. Absorption coefficient $a$ as a function of wavelength $\lambda$. Baikal data taken in spring 2001 (curve 1 -April 4 at $1000 \mathrm{~m}$ depth, curve 2-April 8 at $1000 \mathrm{~m}$ depth and curve 3-May 4 at $1200 \mathrm{~m}$ depth) are compared to data from distilled water [27] ( 4 in the figure) and to cleanest sea water [28] (5 in the figure).

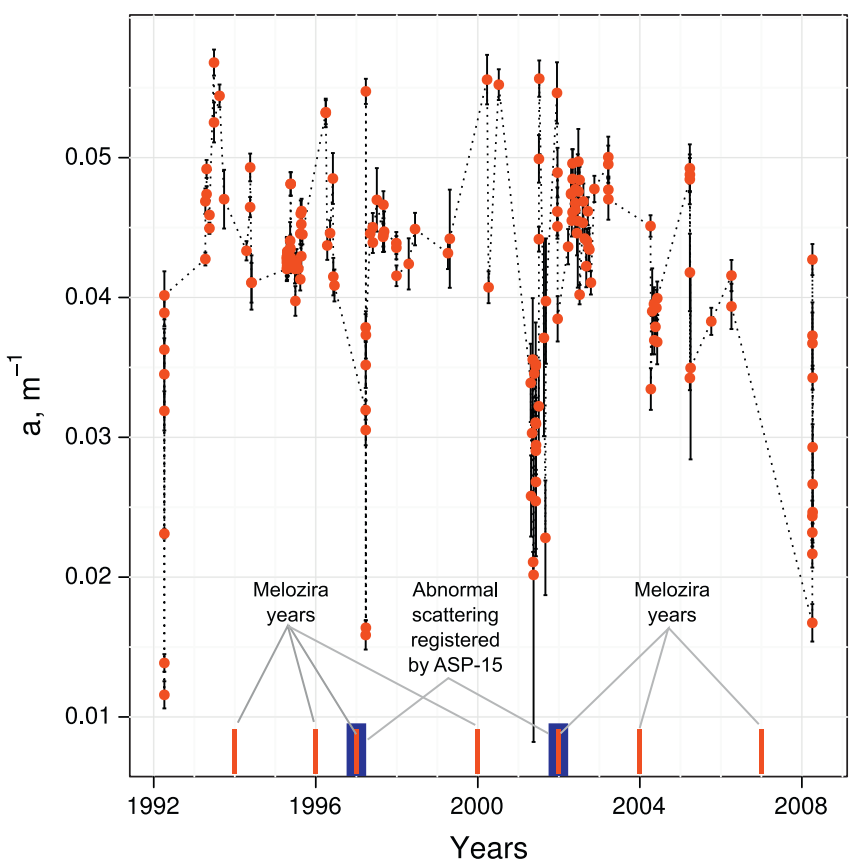

Fig. 7. Long-term behaviour of the absorption coefficient as measured between 1992 and 2008 at wavelength of $488 \mathrm{~nm}$. Marks indicate periods of anomalously increased production of the seaweed Melozira Baikalensis.

Examples of scattering spectra at a depth of $800 \mathrm{~m}$ are presented in Fig. 9. At this depth we observed daily variations in the scattering coefficient of a factor of 4 for some days. Spectra for scattering into the forward hemisphere are usually smooth. Sometimes, however, we observed local extrema in scattering spectra as well.

Variations in the scattering coefficient during the years 1997 and 2002 at depths of $850 \mathrm{~m}$ and $1000 \mathrm{~m}$ are illustrated in Fig. 10. In these years, an anomalously big production of the seaweed

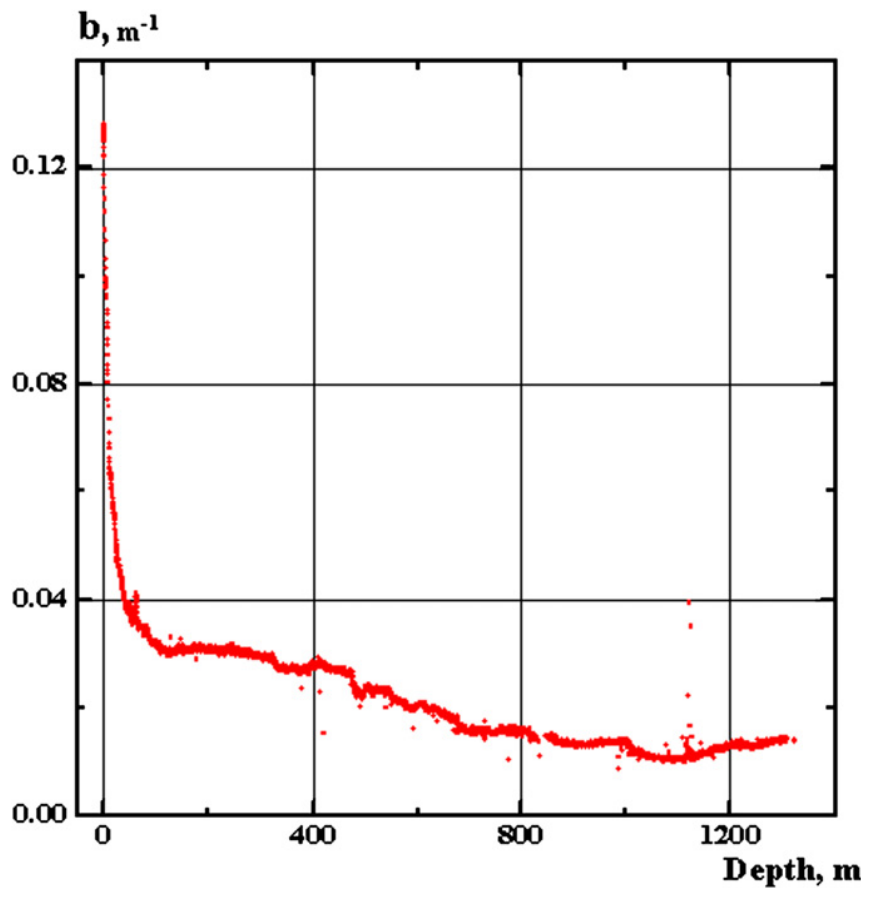

Fig. 8. The depth profile of the forward scattering coefficient $b_{\mathrm{fwd}}(488 \mathrm{~nm})$, as measured by ASP-15 near the NT200 site on March 3, 2000.

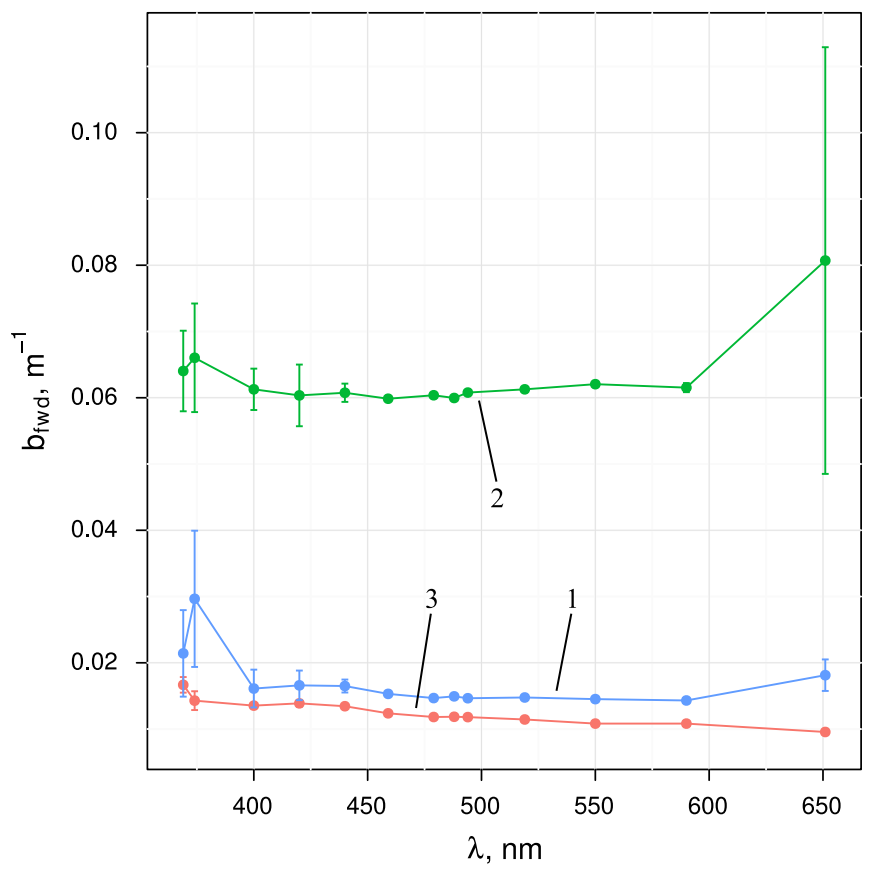

Fig. 9. Spectral dependence on the forward scattering coefficient $b_{\mathrm{fwd}}$, measured near the NT200 site at $800 \mathrm{~m}$ depth in spring 2001. Curve 1-April 4, 2-April 6, 3-April 8.

Melozira Baikalensis was observed. In both cases (Fig. 10, left and right panels) the forward scattering coefficient changed by nearly a factor of 10 . We note that variations in the absorption coefficient were very small at the same time in both years.

The asymmetry coefficient $K(\lambda)$ of the scattering phase function varies from 5 to 10 in deep water and from several tens to several hundreds units in surface water. It is related to the size distribution of suspended particles. From time to time, also the dependence of the asymmetry coefficient on the wavelength $\lambda$ shows narrow extrema. 

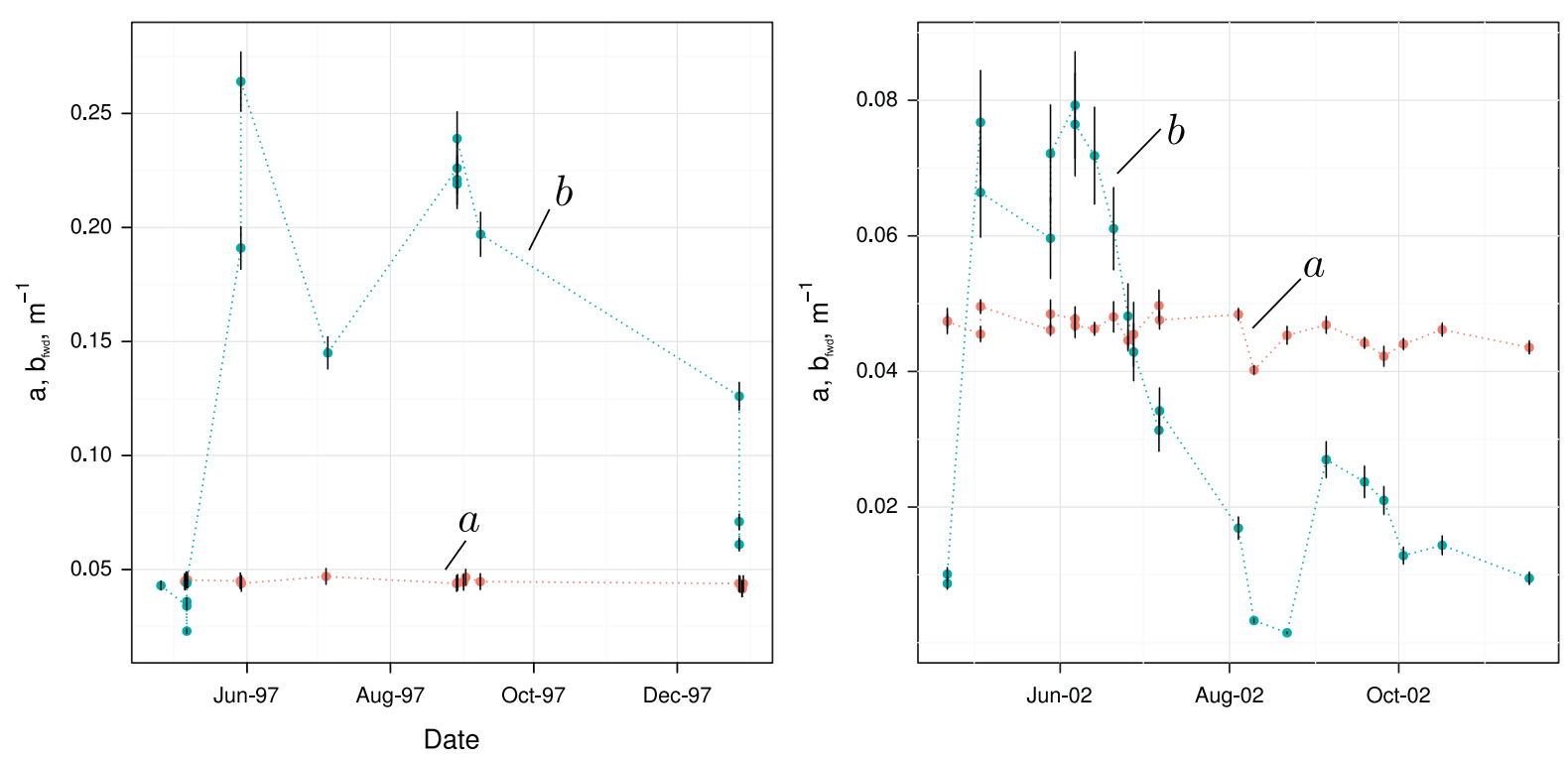

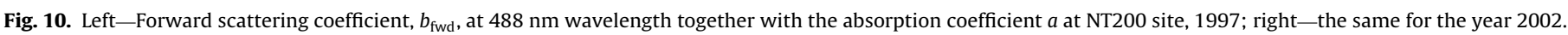

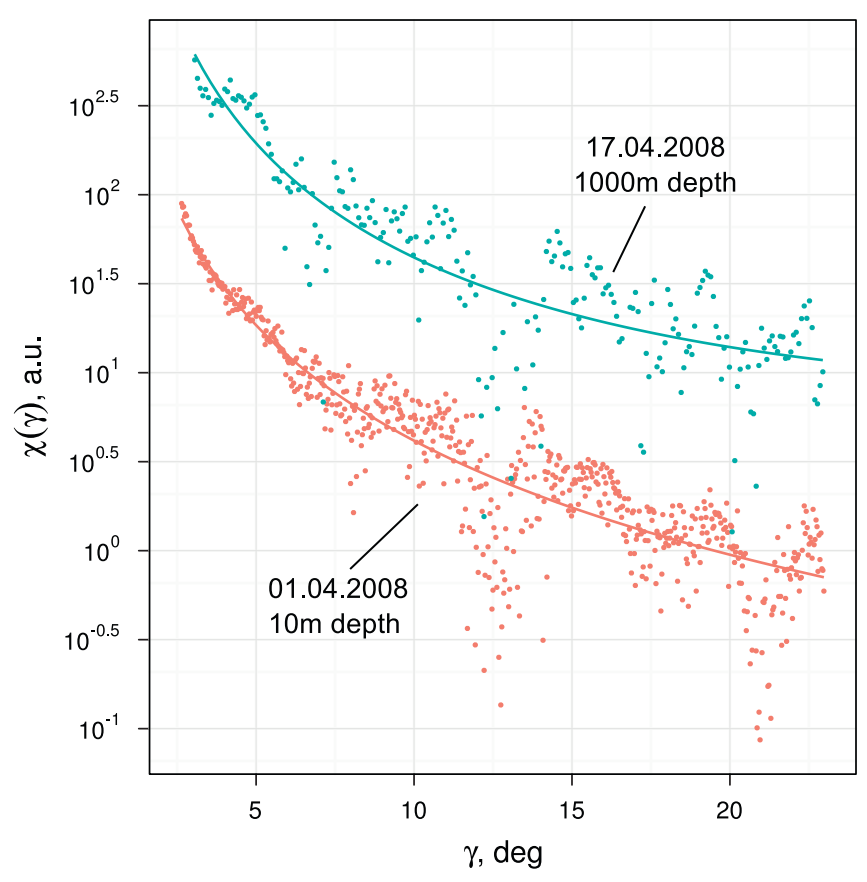

Fig. 11. Top curve-example of scattering phase function for white light (arbitrary units), NT200 site, $1000 \mathrm{~m}$ depth, 2008; bottom curve-the same for surface water near NT200 site, 2007.

\subsection{Scattering phase function}

The scattering phase function for deep water in Lake Baikal is very forward-peaked - similar to clean sea water. Fig. 11 shows the scattering phase function (not normalized) for deep (top curve) and shallow (bottom curve) water. We used white light in order to be sensitive at large angles. Such an approach is justified, because the range of suspended particles sizes $\left(10^{-7}-10^{-5} \mathrm{~m}\right)$, which are liable for light scattering, is substantially wider than the wavelength window of visible light, and since the form of the phase function should only weakly depend on the wavelength (with the exception of interference structures). Our measurements of the phase function, using different interference light filters, from 2 to $10^{\circ}$, confirm this assumption.

The bottom curve in Fig. 11 decreases much faster with the angle than the top one, since the size of suspended material in surface layers is larger than in the deeper ones.

It is difficult to measure the scattering function at angles less than $2.5^{\circ}$ with ASP-15, because direct light dominates in this region. Therefore a direct determination of the average cosine of the scattering angle, $\overline{\cos \gamma}$, is problematic. An exponential extrapolation of the scattering phase function towards the small angle region was used for the estimations of the average cosine of scattering angles. ${ }^{1}$ The characteristic value of $\overline{\cos \gamma}$ is about 0.9 for Baikal water at $1000 \mathrm{~m}-1200 \mathrm{~m}$ depth.

The important parameter for interpreting results of underwater neutrino telescopes is the so-called effective scattering length, $L_{b}$ (eff). The effective scattering length is defined as

$L_{b}(\mathrm{eff})=L_{b} /(1-\overline{\cos \gamma})$.

Actually there is a method to determine absorption length and effective scattering length with the help of the neutrino telescope itself. We have performed a corresponding experiment in 1997 [30] where we illuminated the photomultipliers of the telescope by a distant, pulsed laser. From the dependence of the measured amplitudes and times on the distance between the laser and various photomultipliers, $L_{a}$ and $L_{b}$ (eff) at $475 \mathrm{~nm}$ wavelength could be determined, with $L_{a}=22 \mathrm{~m}$ and $L_{b}$ (eff) $=480 \mathrm{~m}$. Assuming $\overline{\cos \gamma}=0.9$, this corresponds to $L_{b}=48 \mathrm{~m}$, which is well in the range of values determined with ASP-15. The agreement between the two complementary methods confirmed our trust in both methods.

\section{Summary and outlook}

We have described the working principle and the design of the stationary underwater device ASP-15 and presented results obtained for the absorption length, the scattering length and

\footnotetext{
${ }^{1}$ Although the different extrapolations of the scattering phase function towards the small angle region can be used, the error of $b_{\text {fwd }}$ caused by using any realistic extrapolation procedure is less than mentioned above errors of $\delta b_{\mathrm{fwd}} \sim 10 \%$ of $b_{\mathrm{fwd}}$ measurements.
} 
the scattering phase function. The interpretation of data from neutrino telescopes operated in open water or lakes requires a continuous monitoring of water parameters, since many of them vary in time, often to a degree which significantly affects the propagation of photons between a light source and the detecting photomultipliers. Transient phenomena with durations of hours to days and in particular seasonal variations at time scales of weeks to months will have to be taken into account when evaluating the data of cubic kilometer telescopes. For these large arrays, with ten or hundred thousands of neutrinos compared to hundreds or thousands for present detectors, systematic errors rather than statistical errors may become the dominant ones. Since commercial devices do not meet the requirements of longterm, remote operation and sufficient precision, we have constructed ASP-15 which has proven to fulfill these requirements and has been operated over long periods. Based on the experience gained with ASP-15, we intend to construct a device which will be able to work down to greater depths, i.e. to be operated at sites of neutrino telescopes in deep oceans. At the same time, we will improve the reliability of the device. This device will be used as a part of site properties monitoring system of the Baikal Gigaton Volume Detector (GVD). For a precise understanding of neutrino data, one should use several complementary methods: (a) direct measurement of absorption length, scattering length and scattering phase function with a device like ASP-15, (b) determination of absorption length and effective scattering length with the help of artificial light sources and the telescope itself, (c) continuous monitoring of possible transient phenomena with the help of the information from reconstructed muon tracks.

\section{Acknowledgements}

This work was supported in part by the Russian Ministry of Education and Science (GK 02.740.11.0018, GK 16.518.11.7056, P1242, P2504, P133, P878, P1146, RNP 2.2.1.1/1483, RNP 2.1.1/ 1539, RNP 2.2.1.1/5901, REC “BAIKAL"), by the German Ministry of Education and Research, by the Russian Foundation for Basic Research (grants 11-02-00983, 11-02-12097).

\section{References}

[1] M.A. Markov, in: Proceedings of the 1960, Annual International Conference on High Energy Physics, Rochester, 1960, p. 578.

[2] J. Babson, et al., Physical Review D 42 (1990) 3613.

[3] I.A. Belolaptikov, et al., Astroparticle Physics 7 (1997) 263.

[4] G.V. Domogatsky, in: Proceedings of the XII International Conference on Neutrino Physics, Sendai, 1986, World Scientific Publications, Singapore, 1986, p. 737.

[5] L.B. Bezrukov, et al., Soviet Journal of Nuclear Physics 52 (1990) 54.

[6] V.A. Balkanov, et al., Astroparticle Physics 12 (1999) 75.

[7] R.I. Bagduev, et al., Nuclear Instruments and Methods in Physics Research Section A 420 (1999) 138.

[8] V. Aynutdinov, et al., Astroparticle Physics 25 (2006) 140

[9] V. Aynutdinov, et al., Nuclear Instruments and Methods in Physics Research Section A 602 (2009) 14.

[10] M. Circella, et al., Nuclear Instruments and Methods in Physics Research Section A 602 (2009) 1.

[11] A. Capone, et al., Nuclear Instruments and Methods in Physics Research Section A 602 (2009) 47.

[12] P.A. Rapidis, Nuclear Instruments and Methods in Physics Research Section A 602 (2009) 54.

[13] U.F. Katz, Nuclear Instruments and Methods in Physics Research Section A 602 (2009) 40.

[14] Zh.-A. Dzhilkibaev, A. Gazizov, C. Spiering, in: E. deWolf (Ed.), Proceedings of the VLVNT Workshop, Amsterdam, 2003, p. 99.

[15] Zh.-A. Dzhilkibaev, B. Shaibonov, Preprint INR-1073/2002, Moscow, 2002 (in Russian).

[16] C.D. Mobley, Academic Press, San Francisco, 1994

[17] A. Capone, et al., Nuclear Instruments and Methods in Physics Research Section A 487 (2002) 423.

[18] H. Bradner, G. Blackinton, Applied Optics 23 (1984) 1009.

[19] H. Bradner, in: L. Resvanis (Ed.), Proc. 2. NESTOR International Workshop, Pylos, Greece, 1992, p. 247.

[20] E.G. Annasontzis, et al., Nuclear Instruments and Methods in Physics Research Section A 349 (1994) 242.

[21] E.G. Anassontzis, et al., Astroparticle Physics 34 (2010) 187.

[22] V.N. Pelevin, Izvestiya of Academy of Sciences, USSR. Atmospheric and Ocean Physic 1 (1965) 539.

[23] V.N. Pelevin, T.M. Prokudina, Ocean and atmospheric optics, Nauka Publisher Leningrad, 1972. (p. 148).

[24] L.B. Bezrukov, et al., Okeanologiya 30 (1990) 1022.

[25] O.N. Gaponeko, R.R. Mirgazov, B.A. Tarashansky, Izvestiya Atmospheric and Oceanic Optics 9 (1996) 677.

[26] N.M. Budnev, et al., Atmospheric and Oceanic Optics 18 (2005) 109.

[27] R.M. Pope, E.S. Fry, Applied Optics 36 (1997) 8710.

[28] V.N. Pelevin, V.V. Rostovseva, Atmospheric and Oceanic Optics 10 (1997) 989

[29] V. Balkanov, et al., Nuclear Instruments and Methods in Physics Research Section A 498 (2003) 231.

[30] I.A. Belolaptikov, et al., Applied Optics 38 (1999) 6818. 